\title{
Altered muscle activation in agility dogs performing warm-up exercises: an acoustic myography study
}

\author{
L.H. Fuglsang-Damgaard ${ }^{1 *}$, A.P. Harrison ${ }^{2}$ and A.D. Vitger ${ }^{3}$ \\ ${ }^{1}$ Hulbækvej 36, 8830 Tjele, Denmark; 2University of Copenhagen, PAS (Physiology), Faculty of Health and Medical Sciences, Dyrlægevej \\ 100, 1870 Frederiksberg C, Denmark; ${ }^{3}$ DVM, University of Copenhagen, Faculty of Health and Medical Sciences, Veterinary Clinical Sciences, \\ Dyrlægevej 16, 1870 Frederiksberg C, Denmark; hoeg@mfd.dk
}

Received: 6 December 2019 / Accepted: 14 July 2020

() 2020 Wageningen Academic Publishers

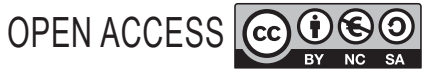

RESEARCH ARTICLE

\begin{abstract}
Agility is physically demanding and dogs encounter a considerable risk of injury during training and competition. Pre-performance warm-up is used routinely among human athletes to prepare the tissues for these physical demands, but in canine sports evidence for effects of warm-up is lacking. The aim of this study was to investigate the effects of warm-up in dogs on two major muscles involved in locomotion. It was hypothesised that, after warm-up, the muscles would be used more efficiently (more fibre resting time/total time), recruit fewer fibres (reduced spatial summation) and/or activated with a lower firing frequency (reduced temporal summation). The following factors 'sex, age, weight, height, training level and agility experience' were evaluated for their potential impact on muscle function parameters. Fourteen large ( $>46 \mathrm{~cm}$ at the withers) agility dogs of different breeds and training levels performed a 5 min warm-up program three times, with a 2 min break between the programs for recording purposes. Acoustic myography sensors were attached on the skin over the muscles $m$. triceps brachii (TB) and m. gluteus superficialis (GS). Recordings of muscle activity were made, while the dogs trotted before warm-up and after each 5 min warm-up program. The dogs used TB more efficiently after $5 \mathrm{~min}(P<0.05), 10 \mathrm{~min}(P<0.05)$ and $15 \mathrm{~min}(P<0.001)$ of exercise compared to pre-warm-up values. No changes were found in the activity of GS. For well-trained dogs, TB recruited fewer muscle fibres after 10 and 15 min of warm-up compared to dogs that trained less than $1 \mathrm{~h}$ weekly $(P<0.03)$. For dogs with more than 2 years of experience, GS had a lower firing frequency before and after 10 min warm-up compared to dogs with less experience. The results indicate that warm-up alters muscle activation by an increased muscular efficiency. Training level and experience have an influence on muscle function parameters.
\end{abstract}

Keywords: canine sport, muscle function, AMG, injury, physical activity

\section{Introduction}

Canine athletes that participate in sports like agility, canicross or flyball, are subjected to intense physical demands and a considerable risk of injury. The risk of having suffered a sports-related injury has been reported to be $33 \%$ for agility dogs (Levy et al., 2009) and 22\% for dogs participating in canicross (Lafuente and Whyle 2018). Warm-up is used extensively among human athletes to improve performance (McGowan et al., 2015) and to reduce the risk of muscular injury (Bishop, 2003). Few studies have evaluated potential effects of warm-up strategies in dogs. Two retrospective surveys evaluated risk factors for injury among agility dogs by owner questionnaires, and here the odds ratio for injury was not affected by the variable warmup (Cullen et al., 2013; Zachary et al., 2014).

The effects of warm-up are mainly attributed to an increase in muscle temperature. Elevated muscle temperature can improve athletic performance as it has been shown in humans to lead to increased muscle metabolism, muscle fibre function and muscle fibre conduction velocity (McGowan et al., 2015). Warm-up may give some protection against strain injuries, as it increases soft tissue flexibility (Petrofsky et al., 2013; Tsolakis and Bogdanis, 2012), and warm muscles can endure higher forces before 
injury (Strickler et al., 1990). A rapid increase in muscle temperature can be obtained in human athletes by 3-5 min of moderate intensity warm-up exercises, and a $2-4{ }^{\circ} \mathrm{C}$ steady state increase is reached after 10-20 min (Racinais et al., 2017). Warm-up is a broad term and in order to avoid misunderstanding as to what precisely is meant by it in this study, we define it as being a low-intensity fixed exercise set of 5 to 15 min duration, consisting of general muscle-loosening exercises as well as performance-specific exercises, carried out before physical performance with the aim of increasing muscle temperature. We use the term warm-up, as it is the generally accepted term for this pre-performance physical activity, although we cannot know if the aim of increased muscle temperature is actually achieved.

By use of acoustic myography (AMG), this study evaluates the active contractions of specific muscles before and after warm-up exercise. The field of muscle contraction recording is one that goes back a number of decades (Barry et al., 1990; Stokes and Blythe, 2001). Indeed, over the years it has had various names, from mechanomyography to accelerometer myography as well as AMG, yet all of these techniques have relied on the measurement of movement vibrations (Alves and Chau, 2011; Beck et al., 2010; Hemmerling et al., 2004; Herda et al., 2010; Qi et al., 2011; Shinohara and Søgaard, 2006; Tian et al., 2010). In recent years, these techniques have been validated and quantified, revealing that mechanomyography amplitude and frequency patterns are useful for examining motorunit recruitment and firing rate during periods of physical activity (Perry et al., 2001), that mechanomyography can be used to accurately estimate isometric elbow flexion force (Youn and Kim, 2010), that these recordings are more sensitive than surface electromyography in terms of the clinical assessment of muscle pain conditions (Madeleine and Arendt-Nielsen, 2005), and that signal parameters correlate closely with motor unit fatigue in active muscles (Barry et al., 1985).

In recent years, a contact transducer with piezoelectric crystals has improved AMG signals (Bartels et al., 2017; Fenger and Harrison, 2017). With this technique, it has become possible to use AMG for accurate and repeatable real-life recordings of muscle work during physical activities of humans, horses and dogs (Bartels et al., 2017; Fenger and Harrison 2017; Harrison, 2017; Harrison et al., 2013, 2018; Pingel et al., 2019; Riis et al., 2013). For a more detailed explanation of AMG and its comparison with surface electromyography, see Harrison (2017). In brief, both techniques are used to measure the recruitment of muscle fibres. While surface electromyography measures the electrical signals that activate muscle fibres, AMG measures the pressure waves generated by active muscle fibre contraction. Both techniques relate spatial summation in a muscle to the amplitude of the recorded signal, and temporal summation of muscle activity to the frequency of the spikes within that recorded signal.

The AMG signal comprises three parameters. The E-parameter (efficiency) assesses muscle fibre contraction time in relation to time of relaxation, thereby representing the efficiency or coordination of the muscular work. The $\mathrm{S}$-parameter (spatial summation) measures the signal amplitude in relation to a full $6 \mathrm{~dB}$ signal equivalent to $1 \mathrm{~V}$, and thus it represents the recruitment of muscle fibres. The T-parameter (temporal summation) measures the number of motor unit firings per second, giving the firing frequency of an active muscle. Each parameter is given a score from $0-10$, where 0 is poor and 10 is optimal. Low scores would be indicative of a subject with poor muscle coordination (E), many recruited fibres (S) with a high firing rate $(\mathrm{T})$. On the other hand, a high E-, $\mathrm{S}$ - and T-score would be measured in subjects with a high degree of muscle coordination (E), few recruited muscle fibres (S) and a low firing rate (T) (Harrison, 2017). Figure 1 shows a recorded AMG signal. It illustrates the E-, $\mathrm{S}$ - and T-parameters in relation to the pressure spikes of the recorded signal, and demonstrates calculations for the E-, S- and T-score. The threshold was routinely set to 0.20 that is to say $19.90 \mathrm{mV}$ and T-max was set to $120 \mathrm{~Hz}$.

The aim of this study was to investigate the effects of warmup on two major muscles involved in canine locomotion and to evaluate if the duration of the warm-up program $(5,10$ or $15 \mathrm{~min}$ ) influenced the results. Additionally, it was evaluated if the muscle function parameters were influenced by the dogs' sex, age, weight, height, training level and agility experience. It was hypothesised that the muscles would show higher E-, S- and/or T-scores as a result of warm-up, irrespective of dog-related factors.

\section{Materials and methods}

\section{Animals}

Dogs of different breeds and over 18 months of age were recruited through social media (Facebook). To ensure the ability of the dogs to wear the harness with the recordingdevice, a minimum height at the withers of $43 \mathrm{~cm}$ was chosen according to the FCI agility regulation (Federation Cynologique Internationale, 2018) for the size of the dogs participating in the class for large dogs. All the dogs had previous agility experience (i.e. the dog could recognise hurdles and an A-frame, and could encounter them with ease). Moreover, all the dogs had to be able to perform agility without any signs of lameness assessed by one of the authors (L.H.F.-D.) in cooperation with an assisting veterinarian. 


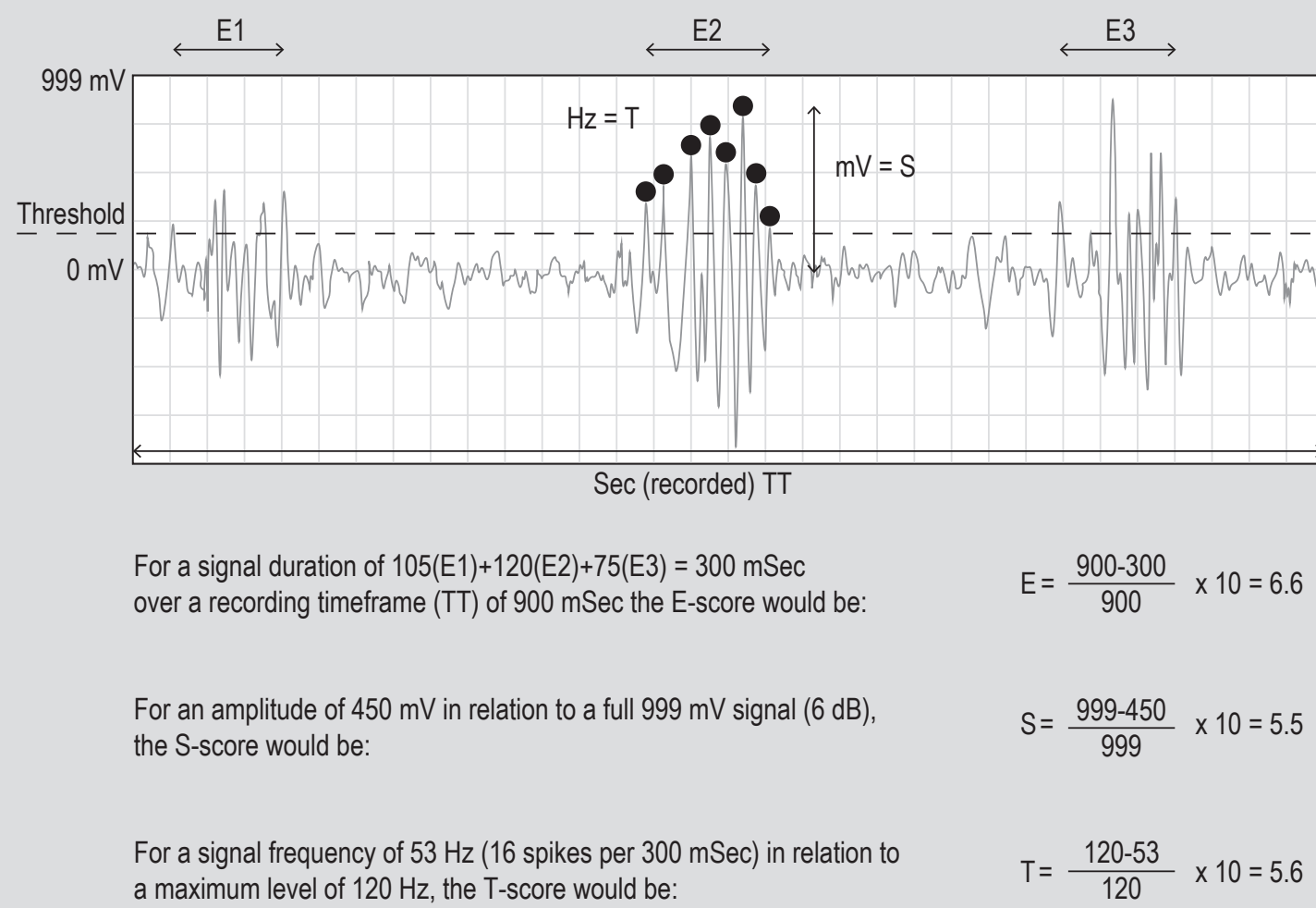

Figure 1. Recorded acoustic myography signal in relation to its E-, S- and T- scores.

\section{Questionnaire}

The dog owners filled out a questionnaire that addressed the topics of; the dog's name, sex, neuter status, date of birth, height over the withers and weight. In addition, the owners were asked to report the dog's health status, agility experience (total number of months) and hours of weekly agility training over the three months prior to this study. The data were used to investigate how dog-related factors influenced muscle activity during the trial.

\section{Study design}

Prior to the warm-up exercises the dogs walked $160 \mathrm{~m}$ from the car park to the training area where the AMG equipment was mounted. The mounting of the AMG equipment and introducing the protocol to the owner took a minimum of 15 min during which period the dog was standing or resting. Total warm-up time was 15 min per dog, divided into three 5 min sessions. The first AMG recording was made before the warm-up exercises began. Hereafter, AMG recordings were made at the end of each 5 min warm-up period, until a total of four AMG recordings had been collected for each dog. Acoustic myography recordings were made while the dog was trotting in a straight line by the owner's side until a clean run of $10 \mathrm{~s}$ had been obtained, with consistent gait and without the dog jumping or shifting pace.
Each 5 min session consisted of 1 min of brisk walk, 1 min of trot, $0.5 \mathrm{~min}$ of canter (estimated average speed around 6, 8 and $>10 \mathrm{~km} / \mathrm{h}$, respectively); 1 minute of shifting position to the left and the right of the handler, 0.5 min of weaving between the owners legs while the owner walked, $0.5 \mathrm{~min}$ of figure-of-eight movements between the owner's legs while the owner stood still, and $0.5 \mathrm{~min}$ of walking the cavaletti. The cavaletti setup for all dogs consisted of 6 consecutive hurdles $0.5 \mathrm{~m}$ apart and at a height of $20 \mathrm{~cm}$. If a dog did not step between each hurdle, a new attempt was allowed. Dogs were offered water and had a 2 min break between each 5 min session.

\section{Acoustic myography recordings}

A commercial CURO device (CURO-Diagnostics ApS, Bagsværd, Denmark; sampling at $4 \mathrm{kHz}$ ) and AMG sensors with a diameter of $50 \mathrm{~mm}$ were used to measure the AMG signals of $m$. triceps brachii (TB) and $m$. gluteus superficialis (GS). The dogs wore a Julius-K9 harness size 0,1 or 2 (Julius-K $9^{\oplus}$, Szigetszentmiklós, Hungary) where the CURO was snugly fitted into the handle (Figure 2A). Two AMG sensors were positioned on the dog's right side; one over GS between the iliac crest and the ischial tuberosity (Figure 2B), and one over the lateral head of $\mathrm{TB}$, between the proximal humerus and the olecranon (Figure 2C). The fur between the sensor and skin was not cut. Acoustic gel (Ekkomarine Medico A/S, Holstebro, Denmark) was placed between the skin and the sensors. The sensors were fastened with the 


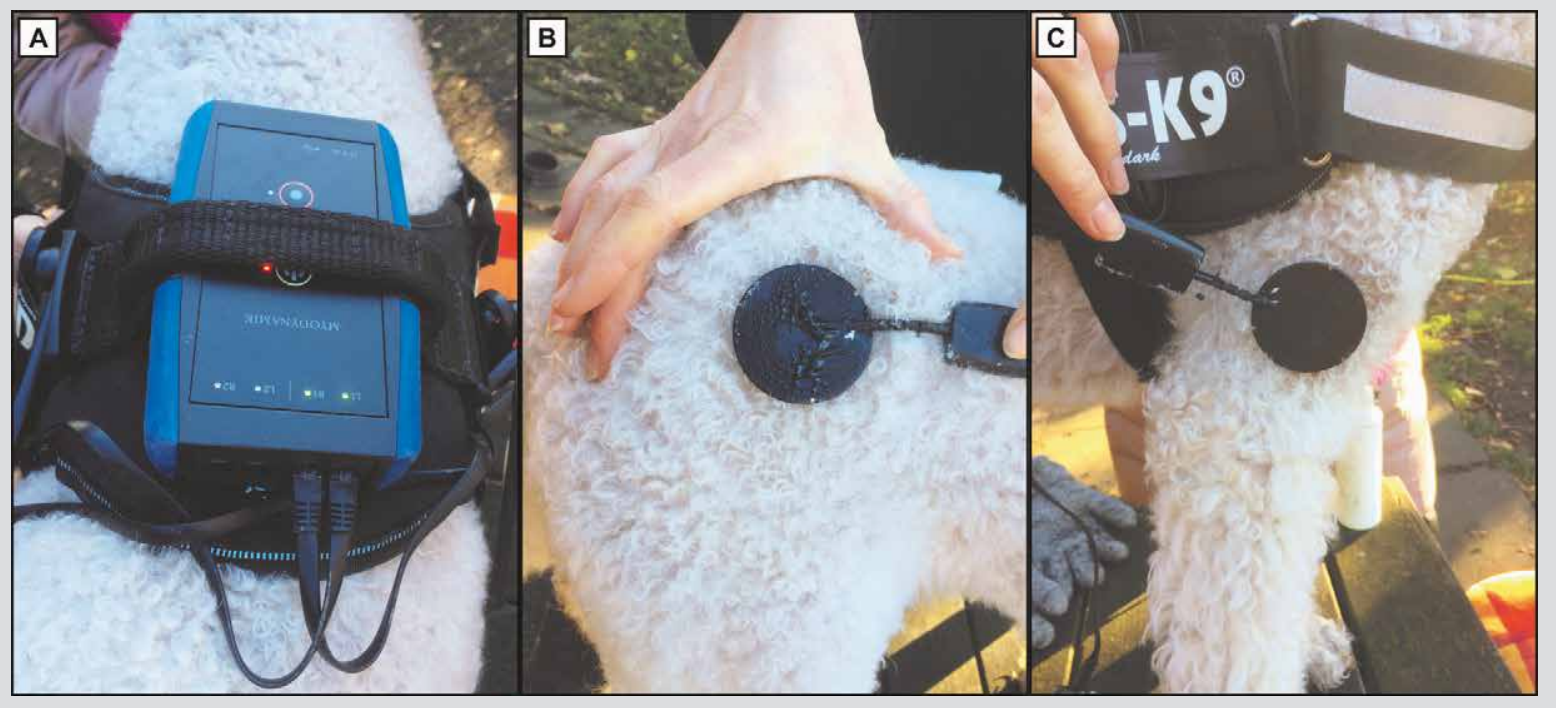

Figure 2. Position of acoustic myography (AMG) equipment on 14 agility dogs performing warm-up exercises. (A) The position of the CURO device under the handle of a Julius-K9 harness; (B) the position of the AMG sensor on $m$. gluteus superficialis between the illiac crest (position of thumb) and the ischial tuberosity (position of middle finger); (C) the position of the AMG sensor on $m$. triceps brachii between the proximal humerus and the olecranon of the ulna.

adhesive bandage Snøgg (Snøgg AS, Kristiansand, Norway) and connected to the CURO device. The equipment was positioned and mounted by one of the authors (L.H.F.-D.) and an assisting veterinarian who had both received prior training.

The muscle signal was transmitted via Wi-Fi from the CURO-device to an i-Pad 2 (Apple Inc., Cupertino, CA, USA) and measurements were followed in real-time to ensure proper transmission of recordings.

\section{Statistical analysis}

The data were initially tested for a normal distribution. Differences between means were tested for statistical significance using GraphPad InStat 3 for Mac (Version 3.0b, 2003; GraphPad Inc., La Jolla, CA, USA). For data with a normal distribution a repeated measures ANOVA was used, otherwise a Friedmans test was applied. The Tukey-Kramer multiple comparisons test was used for the post hoc tests. Differences between means with a $P$-value of $<0.05$ were considered significant.

The effects of sex, age, weight, height, training level and agility experience of the individual dogs were tested by clustering the dogs into two groups for each characteristic. Differences between means were tested for statistical significance using Real Statistic Resource Pack for Excel 2010/2013/2016 for Windows (version 4.13, 2010, Real Statistics Using Excel, Charles Zaiontz) to perform a Wilcoxon Rank-Sum test.

\section{Results}

Fourteen dogs ( 8 bitches, 6 males) of different breeds were recruited. All of the recruited dogs participated in the study and completed it successfully. The dogs had a mean age of $4.4 \pm 2.3$ years. Their mean weight and height at the withers were $22.6 \pm 6.3 \mathrm{~kg}$ and $55.9 \pm 5.5 \mathrm{~cm}$, respectively, and all the dogs had a body condition score of 4-5 out of 9 (Laflamme, 1997). An overview of the dogs that participated in the study can be found in Table 1 .

Table 2 presents the distribution of the 14 dogs into one of two groups according to their sex, age, weight, height, training level and agility experience, when testing if these factors influenced muscle parameters before or after warmup.

\section{Effect of warm-up on study group (14 dogs)}

\section{m. triceps brachii}

Compared to baseline, the mean E-score of all dogs increased after 5 min warm-up by $38 \%$ ( $P=0.018)$, after $10 \mathrm{~min}$ warm-up by $44 \%(P=0.025)$ and after $15 \mathrm{~min}$ warm-up by $56 \%(P=0.0002)$. The differences between warm-up sessions were not significant. The mean S-score changed after 5 min warm-up by $9 \%(P=0.770)$, after 10 min warm-up by $17 \%(P=0.241)$ and after $15 \mathrm{~min}$ warm-up by $17 \%(P=0.060)$. The mean $\mathrm{T}$-score only changed $1-4 \%$ after warm-up. The results for $\mathrm{S}$ - and T-scores were not significant. Figure $3 \mathrm{~A}-\mathrm{C}$ show a graphic presentation of the results for TB. 
Table 1. Descriptive data of 14 agility dogs performing warm-up exercises.

\begin{tabular}{|c|c|c|c|c|c|c|}
\hline Breed & Sex and neuter status ${ }^{1}$ & $\begin{array}{l}\text { Age } \\
\text { (years) }\end{array}$ & $\begin{array}{l}\text { Height (to withers) } \\
\text { (cm) }\end{array}$ & $\begin{array}{l}\text { Weight } \\
(\mathrm{kg})\end{array}$ & $\begin{array}{l}\text { Experience } \\
\text { (years) }\end{array}$ & $\begin{array}{l}\text { Training level } \\
\text { (hours per week) }\end{array}$ \\
\hline Shetland sheepdog & $\mathrm{Mn}$ & 3.0 & 46 & 13 & 2.0 & $2-3$ \\
\hline Border collie & $\mathrm{F}$ & 3.0 & 49 & 18 & 2.5 & $<1$ \\
\hline Border collie & M & 1.5 & 52 & 18 & 1.0 & $2-3$ \\
\hline Border collie & $\mathrm{F}$ & 8.0 & 55 & 18 & 7.0 & $<1$ \\
\hline Tervueren & $\mathrm{F}$ & 3.0 & 59 & 18 & 2.0 & $<1$ \\
\hline Tervueren & $\mathrm{F}$ & 4.5 & 56 & 20 & 3.0 & $<1$ \\
\hline Tervueren & $\mathrm{F}$ & 6.5 & 62 & 22 & 2.0 & $<1$ \\
\hline Australian shepherd & $\mathrm{F}$ & 2.0 & 50 & 18 & 1.0 & $1-2$ \\
\hline Australian shepherd & M & 8.0 & 57 & 25 & 7.5 & $<1$ \\
\hline Golden retriever & M & 4.5 & 55 & 28 & 2.0 & $1-2$ \\
\hline Old English Sheepdog & $\mathrm{Fn}$ & 8.0 & 61 & 36 & 6.0 & $1-2$ \\
\hline Labradoodle & $\mathrm{Fn}$ & 4.5 & 64 & 29 & 0.5 & $<1$ \\
\hline Mixed breed & $\mathrm{Mn}$ & 2.5 & 54 & 23 & 1.5 & $1-2$ \\
\hline Mixed breed & $\mathrm{Mn}$ & 2.5 & 63 & 30 & 0.2 & $<1$ \\
\hline
\end{tabular}

Table 2. The post hoc grouping according to characteristics of 14 agility dogs performing warm-up. Values in brackets represent the number of subjects.

\begin{tabular}{lll} 
Character & & \\
\hline Sex & Male $(6)$ & Bitch $(8)$ \\
Age & $\leq 3$ years $(7)$ & $>3$ years $(7)$ \\
Height & $\leq 55 \mathrm{~cm}(7)$ & $>55 \mathrm{~cm}(7)$ \\
Weight & $\leq 21 \mathrm{~kg}(7)$ & $>21 \mathrm{~kg}(7)$ \\
Agility experience & $<2$ years $(5)$ & $\geq 2$ years $(9)$ \\
Training level & $<1 \mathrm{~h}$ a week $(8)$ & $\geq 1$ h a week $(6)$
\end{tabular}

\section{m. gluteus superficialis}

No effect of warm-up was found for the mean E-, S- or T-scores measured on GS. For this muscle, the maximum change in any parameter from baseline was seen in the $\mathrm{S}$-score with 13\% ( $P=0.371)$ increase after 15 min of warm-up. Figure 3D-E show a graphic presentation of the results for GS.

Corresponding data for TB and GS can be found in Supplementary Table S1.

\section{Effects of dog-related factors}

m. triceps brachii

As visualised in Table 3, dogs that trained for $1 \mathrm{~h}$ a week or more, had a higher mean S-score for TB after 10 and 15 min of warm-up compared to dogs that trained less than $1 \mathrm{~h}$ per week ( $10 \mathrm{~min}$ : training $>1 \mathrm{hr}, \mathrm{S}=5.7$, training $<1 \mathrm{hr}, \mathrm{S}=2.6, P=0.020 ; 15$ min: training $>1 \mathrm{~h}, \mathrm{~S}=5.3$, training $<1 \mathrm{~h}, \mathrm{~S}=2.8, P=0.028)$. Dogs with a height at the withers of above $55 \mathrm{~cm}$, had a lower mean S-score after $15 \mathrm{~min}$ of warm-up compared to dogs with a height of $55 \mathrm{~cm}$ or below (height $\leq 55 \mathrm{~cm}: \mathrm{S}=3.00$, height $\leq 55 \mathrm{~cm}: \mathrm{S}=4.86, P=0.035$ ) as shown in Table 4. No difference was found for sex, age, weight or experience.

m. gluteus superficialis

As visualised in Table 5, dogs with two years of agility experience or more had a higher mean $\mathrm{T}$-score before and after 10 min of warm-up compared to dogs with less than two years of experience (Before: experience $>2$ years, $\mathrm{T}=8.9$, experience $<2$ years, $\mathrm{T}=7.0, P=0.020 ; 10 \mathrm{~min}$ : experience $>2$ years, $\mathrm{T}=8.7$, experience $<2$ years, $\mathrm{T}=7.6, P=0.046$ ). No difference was found for sex, age, weight, height or training level.

Corresponding data for TB and GS grouped according to each dog-related factor tested can be found in Supplementary Tables S2-S7.

\section{Discussion}

By the use of AMG this study has documented altered muscle activation in dogs after warm-up. E-scores for TB increased after the performance of warm-up exercise, while the hypothesis that both muscles would show higher scores as a result of warm-up has been rejected for GS. The E-score measures the time during which muscle fibres are actively contracting compared to the time of relaxation. 
Effect of pre-exercise physical activity on $m$. triceps brachii
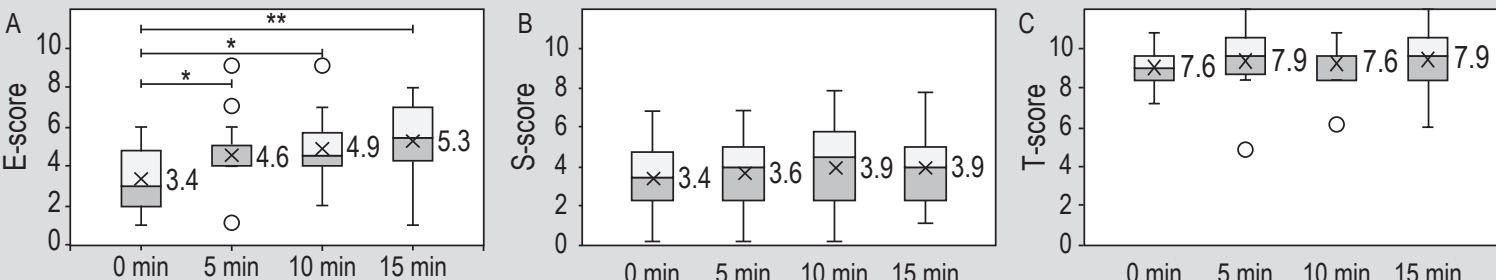

Effect of pre-exercise physical activity on $m$. gluteus superficialis
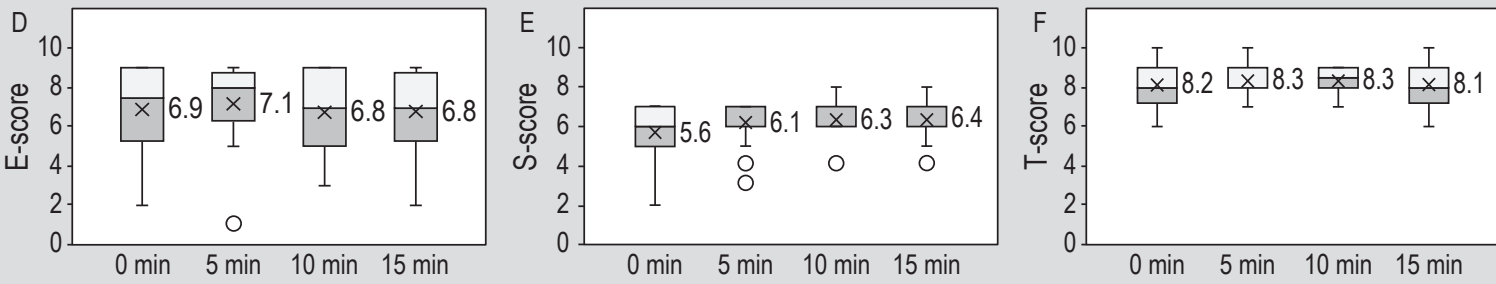

Figure 3. Effect of time spent on warm-up exercises measured on 14 large (height at the withers above $43 \mathrm{~cm}$ ) agility dogs with the use of acoustic myography recordings of $m$. triceps brachii and $m$. gluteus superficialis. The boxplots present the mean recorded score for fibre resting time/total time (E-score), spatial summation (S-score), temporal summation (T-score) for $m$. triceps brachii $(A-C)$ and $m$. gluteus superficialis (D-F) measured while trotting before performing warm-up exercise and after 5,10 and 15 min of warm-up exercises, respectively. Significant differences are noted ${ }^{*} P<0.05$, ${ }^{* *} P<0.001$. The E-score for $m$. triceps brachii increased after $5(P=0.018), 10(P=0.025)$ and $15(P=0.0002) \mathrm{min}$ of warm-up exercises compared with the score measured pre-warm-up. No effect of warm-up exercise was found for $m$. gluteus superficialis.

Table 3. The average recorded score and standard deviation for fibre resting time/total time ${ }^{1}$ (E-score), spatial summation ${ }^{1}$ (S-score), temporal summation ${ }^{1}$ (T-score) for $m$. triceps brachii and $m$. gluteus superficialis on 14 large agility dogs ${ }^{2}$ performing warm-up exercises. ${ }^{3,4}$

Training level m. triceps brachii

$$
0 \min
$$

$$
5 \min
$$

m. gluteus superficialis

$0 \min \quad 5 \min \quad 10 \mathrm{~min} \quad 15 \mathrm{~min}$

\begin{tabular}{|c|c|c|c|c|c|c|c|c|c|c|c|c|c|c|c|c|}
\hline$\geq 1$ h weekly & 3.50 & \pm 1.50 & 5.00 & \pm 2.52 & 5.00 & \pm 1.53 & 5.33 & \pm 2.36 & 6.00 & \pm 1.63 & 6.83 & \pm 1.57 & 5.67 & \pm 1.70 & 6.00 & 4.00 \\
\hline$<1 \mathrm{~h}$ weekly & 3.25 & \pm 1.39 & 4.38 & \pm 1.41 & 4.75 & \pm 1.98 & 5.25 & \pm 1.98 & 8.00 & 1.00 & 8.00 & 1.75 & 9.00 & 3.25 & 6.88 & \pm 2.20 \\
\hline$P$-value & 0.796 & & 0.846 & & 0.747 & & 0.846 & & 0.093 & & 0.333 & & 0.156 & & 0.699 & \\
\hline \multicolumn{17}{|l|}{ S-score } \\
\hline$\geq 1$ h weekly & 4.00 & \pm 0.00 & 4.83 & \pm 1.77 & 5.67 & \pm 1.25 & 5.33 & \pm 1.37 & 6.00 & \pm 0.82 & 6.00 & \pm 1.15 & 7.00 & 3.00 & 7.00 & 2.25 \\
\hline$<1$ h weekly & 2.38 & \pm 1.80 & 2.75 & \pm 1.79 & 2.63 & \pm 1.93 & 2.88 & \pm 1.54 & 5.38 & \pm 1.80 & 7.00 & 1.00 & 6.50 & \pm 1.12 & 6.50 & \pm 0.87 \\
\hline$P$-value & 0.121 & & 0.093 & & 0.020 & & 0.028 & & 0.796 & & 0.651 & & 0.699 & & 0.846 & \\
\hline \multicolumn{17}{|l|}{ T-score } \\
\hline$\geq 1$ h weekly & 7.17 & \pm 0.69 & 7.50 & 1.50 & 7.83 & \pm 0.69 & 7.67 & \pm 0.75 & 7.83 & \pm 1.07 & 8.00 & \pm 0.58 & 8.33 & \pm 0.75 & 8.00 & \pm 1.00 \\
\hline$<1$ h weekly & 7.88 & \pm 1.05 & 9.00 & 3.75 & 7.50 & \pm 1.12 & 8.00 & \pm 1.41 & 8.50 & \pm 1.41 & 8.50 & \pm 1.00 & 7.00 & 1.00 & 8.25 & \pm 1.20 \\
\hline$P$-value & 0.220 & & 0.366 & & 0.747 & & 0.401 & & 0.366 & & 0.302 & & 0.897 & & 0.561 & \\
\hline
\end{tabular}

E-score

${ }^{1}$ Measured with acoustic myography.

${ }^{2}$ Height at the withers above $43 \mathrm{~cm}$.

${ }^{3}$ The dogs were measured while trotting before performing warm-up exercise and after 5, 10 and 15 min of warm-up exercises, respectively.

${ }^{4}$ The dogs were divided into two groups according to their training level, dogs that trained one $h$ a week or more $(n=6)$ and dogs that trained less than an h per week $(n=8)$ respectively. The groups were tested against each other with a Wilcoxon Rank-Sum test with a $P$-value above 0.05 considered significant. Values in italics were found to be non-parametric and therefore represent the median and the IQR. 
Table 4. The average recorded score and standard deviation for fibre resting time/total time ${ }^{1}$ (E-score), spatial summation ${ }^{1}$ (S-score), temporal summation ${ }^{1}$ (T-score) for $m$. triceps brachii and $m$. gluteus superficialis on 14 large agility dogs ${ }^{2}$ performing warm-up exercises. ${ }^{3,4}$

\begin{tabular}{|c|c|c|c|c|c|c|c|c|c|c|c|c|c|c|c|c|}
\hline \multirow[t]{2}{*}{ Dog height } & \multicolumn{8}{|c|}{ m. triceps brachii } & \multicolumn{8}{|c|}{ m. gluteus superficialis } \\
\hline & \multicolumn{2}{|l|}{$0 \mathrm{~min}$} & \multicolumn{2}{|l|}{$5 \mathrm{~min}$} & \multicolumn{2}{|l|}{$10 \mathrm{~min}$} & \multicolumn{2}{|l|}{$15 \mathrm{~min}$} & \multicolumn{2}{|l|}{$0 \mathrm{~min}$} & \multicolumn{2}{|l|}{$5 \mathrm{~min}$} & \multicolumn{2}{|l|}{$10 \mathrm{~min}$} & \multicolumn{2}{|c|}{$15 \mathrm{~min}$} \\
\hline \multicolumn{17}{|l|}{ E-score } \\
\hline$\leq 55 \mathrm{~cm}$ & 3.14 & \pm 1.35 & 4.43 & \pm 1.81 & 4.86 & \pm 1.35 & 5.29 & \pm 2.36 & 6.57 & \pm 1.72 & 7.43 & \pm 1.51 & 6.86 & \pm 2.12 & 7.43 & \pm 1.72 \\
\hline$>55 \mathrm{~cm}$ & 3.57 & \pm 1.72 & 4.86 & \pm 2.41 & 4.86 & \pm 2.41 & 5.29 & \pm 2.29 & 8.00 & 1.50 & 8.00 & 3.00 & 9.00 & 4.50 & 6.14 & \pm 2.34 \\
\hline$P$-value & 0.609 & & 0.798 & & 0.749 & & 1.000 & & 0.307 & & 1.000 & & 0.798 & & 0.277 & \\
\hline \multicolumn{17}{|l|}{ S-score } \\
\hline$\leq 55 \mathrm{~cm}$ & 4.29 & \pm 1.11 & 4.43 & \pm 1.51 & 5.00 & \pm 1.15 & 5.00 & 1.00 & 5.86 & \pm 0.69 & 6.00 & \pm 1.15 & 6.00 & \pm 1.41 & 6.14 & \pm 1.21 \\
\hline$>55 \mathrm{~cm}$ & 2.43 & \pm 2.51 & 2.86 & \pm 2.48 & 2.86 & \pm 2.79 & 3.00 & \pm 2.45 & 5.43 & \pm 2.15 & 7.00 & 1.00 & 7.00 & 0.50 & 6.57 & \pm 0.98 \\
\hline$P$-value & 0.085 & & 0.160 & & 0.074 & & 0.035 & & 0.749 & & 0.443 & & 0.443 & & 0.609 & \\
\hline \multicolumn{17}{|l|}{ T-score } \\
\hline$\leq 55 \mathrm{~cm}$ & 7.57 & \pm 0.98 & 8.00 & 1.00 & 8.00 & 0.50 & 7.00 & 1.00 & 8.14 & \pm 1.35 & 7.86 & \pm 0.69 & 8.43 & \pm 0.79 & 7.71 & \pm 1.25 \\
\hline$>55 \mathrm{~cm}$ & 7.57 & \pm 1.13 & 9.00 & 5.50 & 7.14 & \pm 1.07 & 7.86 & \pm 1.46 & 8.29 & \pm 1.50 & 8.71 & \pm 0.95 & 9.00 & 1.50 & 8.57 & \pm 0.98 \\
\hline$P$-value & 0.949 & & 0.250 & & 0.074 & & 0.609 & & 0.848 & & 0.085 & & 0.565 & & 0.160 & \\
\hline \multicolumn{17}{|c|}{${ }^{1}$ Measured with acoustic myography. } \\
\hline \multicolumn{17}{|c|}{${ }^{2}$ Height at the withers above $43 \mathrm{~cm}$. } \\
\hline \multicolumn{17}{|c|}{$\begin{array}{l}{ }^{3} \text { The dogs were measured while trotting before performing warm-up exercise and after } 5,10 \text { and } 15 \text { min of warm-up exercises, respectively. } \\
{ }^{4} \text { The dogs were divided into two groups according to their height at the withers, dogs with a height of } 55 \mathrm{~cm} \text { or below ( } \mathrm{n}=7 \text { ) and dogs with a height } \\
\text { above } 55 \mathrm{~cm}(\mathrm{n}=7) \text { respectively. The groups were tested against each other with a Wilcoxon Rank-Sum test with a } P \text {-value above } 0.05 \text { considered } \\
\text { significant. Values in italics were found to be non-parametric and therefore represent the median and the IQR. }\end{array}$} \\
\hline
\end{tabular}

Table 5. The average recorded score and standard deviation for fibre resting time/total time ${ }^{1}$ (E-score), spatial summation ${ }^{1}$ (S-score), temporal summation ${ }^{1}$ (T-score) for $m$. triceps brachii and $m$. gluteus superficialis on 14 large agility dogs ${ }^{2}$ performing warm-up exercises. ${ }^{3,4}$

\begin{tabular}{|c|c|c|c|c|c|c|c|c|c|c|c|c|c|c|c|c|}
\hline \multirow[t]{2}{*}{ Experience } & \multicolumn{8}{|c|}{ m. triceps brachii } & \multicolumn{8}{|c|}{ m. gluteus superficialis } \\
\hline & \multicolumn{2}{|l|}{$0 \mathrm{~min}$} & \multicolumn{2}{|l|}{$5 \mathrm{~min}$} & \multicolumn{2}{|l|}{$10 \mathrm{~min}$} & \multicolumn{2}{|l|}{$15 \mathrm{~min}$} & \multicolumn{2}{|l|}{$0 \mathrm{~min}$} & \multicolumn{2}{|l|}{$5 \mathrm{~min}$} & \multicolumn{2}{|l|}{$10 \mathrm{~min}$} & \multicolumn{2}{|c|}{$15 \mathrm{~min}$} \\
\hline \multicolumn{17}{|l|}{ E-score } \\
\hline$<2$ years & 3.00 & 1.50 & 4.00 & 2.00 & 4.60 & \pm 2.06 & 5.20 & \pm 2.06 & 5.80 & \pm 2.35 & 5.80 & \pm 1.27 & 5.60 & \pm 2.13 & 6.00 & \pm 1.64 \\
\hline$\geq 2$ years & 3.22 & \pm 0.89 & 5.22 & \pm 1.52 & 5.00 & \pm 1.67 & 5.33 & \pm 2.77 & 9.00 & 3.50 & 8.00 & 1.50 & 9.00 & 4.00 & 7.22 & \pm 2.74 \\
\hline$P$-value & 0.505 & & 0.072 & & 0.790 & & 0.947 & & 0.205 & & 0.205 & & 0.205 & & 0.424 & \\
\hline \multicolumn{17}{|l|}{ S-score } \\
\hline$<2$ years & 2.20 & \pm 2.12 & 2.80 & \pm 2.20 & 3.40 & \pm 2.39 & 3.20 & \pm 2.12 & 5.00 & 1.50 & 5.80 & \pm 1.32 & 5.80 & \pm 1.01 & 5.80 & \pm 0.50 \\
\hline$\geq 2$ years & 4.00 & \pm 1.64 & 4.11 & \pm 1.92 & 4.22 & \pm 2.41 & 4.33 & \pm 1.64 & 6.00 & 2.50 & 7.00 & 1.00 & 7.00 & 0.50 & 7.00 & 1.00 \\
\hline$P$-value & 0.110 & & 0.286 & & 0.549 & & 0.286 & & 0.424 & & 0.386 & & 0.463 & & 0.317 & \\
\hline \multicolumn{17}{|l|}{ T-score } \\
\hline$<2$ years & 7.00 & \pm 1.05 & 7.80 & \pm 1.69 & 8.00 & 0.50 & 8.00 & 1.00 & 7.00 & \pm 0.93 & 8.00 & 0.50 & 7.60 & \pm 0.50 & 7.00 & 1.50 \\
\hline$\geq 2$ years & 7.89 & \pm 0.71 & 7.89 & \pm 0.84 & 7.56 & \pm 0.45 & 8.00 & \pm 0.55 & 9.00 & 2.00 & 8.33 & \pm 1.30 & 9.00 & 1.00 & 8.44 & \pm 0.89 \\
\hline$P$-value & 0.125 & & 0.549 & & 0.790 & & 0.386 & & 0.020 & & 0.739 & & 0.046 & & 0.162 & \\
\hline $\begin{array}{l}{ }^{2} \text { Height at th } \\
{ }^{3} \text { The dogs } w \\
{ }^{4} \text { The dogs } w \\
\text { and dogs with } \\
P \text {-value abov }\end{array}$ & $\begin{array}{l}2 \text { years } \\
0.05\end{array}$ & $\begin{array}{l}\text { sured wh } \\
\text { led into tv } \\
\text { onsiderec }\end{array}$ & $\begin{array}{l}\text { ho group } \\
\text { hile trottin } \\
\text { arience or } \\
\text { d signific }\end{array}$ & $\begin{array}{l}\text { ng before } \\
\text { ps accorc } \\
\text { r more ( } \mathrm{n} \\
\text { cant. Valu }\end{array}$ & $\begin{array}{l}\text { e perform } \\
\text { ding to th } \\
\text { n=9) resp } \\
\text { ues in ital }\end{array}$ & $\begin{array}{l}\text { ning warr } \\
\text { heir years } \\
\text { pectively. } \\
\text { alics were }\end{array}$ & $\begin{array}{l}\text { m-up ex } \\
\text { s of expe } \\
\text { The gro } \\
\text { found } t\end{array}$ & $\begin{array}{l}\text { ercise a } \\
\text { erience } \\
\text { oups we } \\
\text { to be no }\end{array}$ & $\begin{array}{l}5,10 \\
\text { ty traini } \\
\text { ed agair } \\
\text { metric a }\end{array}$ & $\begin{array}{l}\text { and } 15 \mathrm{~m} \\
\text { ng, dogs } \\
\text { nst each } \\
\text { and there }\end{array}$ & $\begin{array}{l}\text { nin of wa } \\
\text { with less } \\
\text { other wit } \\
\text { fore repr }\end{array}$ & $\begin{array}{l}\text { arm-up ex } \\
\text { s than tw } \\
\text { th a Wilc } \\
\text { resent the }\end{array}$ & $\begin{array}{l}\text { xercises } \\
\text { no years } \\
\text { coxon Ra } \\
\text { ne media }\end{array}$ & $\begin{array}{l}\text {, respect } \\
\text { of exper } \\
\text { ank-Sum } \\
\text { in and the }\end{array}$ & $\begin{array}{l}\text { tively. } \\
\text { rience } \\
\text { test } \mathrm{W}\end{array}$ & \\
\hline
\end{tabular}


An increased E-score after warm-up means that, for the same physical activity, fibre contraction time has decreased, indicating that muscular work has become more efficient. This effect on muscle fibre activation is in line with other documented effects of warm-up, such as facilitation of muscle contractions and increased transmission speed of nerve impulses (Woods et al., 2007). An increase in efficiency could be expected to drive a muscle to reduce the number of recruited fibres (lower spatial summation), as argued by Bartels et al. (2017). A small sample size may be the reason why we did not see this concomitant change in fibre recruitment, as the results for TB showed only a tendency of increased S-scores (up to $17 \%$ increase). Before warm-up TB elicited fairly low E- and S-scores $(<4)$, while the T-scores were already high $(>7)$ and would therefore be less likely to change significantly after the exercises.

During locomotion, TB extends the elbow, stabilises the leg and drives the dog forward in the stance phase. In this study the sensor was positioned over the lateral head of TB, but it may also have measured some activity from the long head of TB. The long head has been found to be the strongest head of this muscle in greyhounds with a power of $58 \mathrm{~W}$ compared to the lateral head with a power of $9 \mathrm{~W}$ (Williams et al., 2008). When the lateral head of TB was too small to cover the whole surface of the sensor, it was preferred to place the sensor in the direction of the long head to maintain measurements from TB without noise from other muscle groups. Thus, the results should be regarded as recordings from the work of both $m$. triceps brachii lateralis and longus. As both heads are active during the warm-up program in this study, this factor is not expected to affect the results.

The mean E-, S- and T-scores for GS were all above 5 before warm-up, and there was no measurable increase in these scores after any length of warm-up. m. gluteus superficialis, together with $m$. gluteus medius, is one of the primary retractor muscles of the hip in dogs (Schilling et al., 2009). It is active during the last $20 \%$ of the swing phase and the first third of the support phase (Schilling et al., 2009), and it acts to brake the swinging limb and assist in propulsion together with $m$. gluteus medius and other retractor muscles of the hip (Deban et al., 2012). It has also been suggested to primarily stabilise the hip (Evans and de Lahunta, 2000). Schilling et al. (2009) showed that the electromyography signals for GS in a trotting dog on flat ground were very modest compared to the signals during incline trotting. Thus, for a sporting dog, merely trotting on flat ground may have been too easy an exercise to elicit measurable effects of warm-up by AMG. It should also be considered, that if GS mainly contributes to stabilisation rather than propulsion, it may not be very susceptible to the effects of warm-up.
Appropriate warm-up can enhance a human athlete's performance (Fradkin et al., 2010; Kilduff et al., 2013; McGowan et al., 2015), and optimising the warm-up protocol is therefore a major concern for athletes and a focus for research. Still, many factors come into play when designing a warm-up protocol, and not least the fact that sports performance is a very broad field, encompassing short high-intensity sprints as well as prolonged endurance races. Increasing muscle temperature pre-performance is particularly beneficial for human athletes who compete in sprints or sustained high-intensity sports (McGowan et al., 2015), and this is likely to apply for sporting dogs as well. The warm-up protocol chosen for this trial was based on some general warm-up principles with the use of popular exercises among agility dog handlers. A typical warm-up protocol contains both a general and a specific dynamic segment. The purpose of the general warm-up segment is to increase muscle temperature and flexibility, while the specific warm-up segment is implemented to prepare muscles for specific movements required for performance (Bishop, 2003; Fradkin et al., 2010; Woods et al., 2007). Stretching was not included in our trial protocol, although traditionally, static stretching has been part of pre-performance warm-up protocols for human athletes (Fradkin et al., 2010). Stretching may reduce risk of muscular injury during physical activity, but it should be used as part of a regular long-term routine (Woods et al., 2007). Used inappropriately, static stretching has been shown to impair subsequent performance (Faigenbaum et al., 2005; Tsolakis and Bogdanis 2012). For the general segment in this trial, the dogs performed a brisk walk, a trot and a gallop. These movements loosen up muscles and fascia to ease the 'slide and glide' between muscles, thereby facilitating a smooth and coordinated force transmission (Carvalhais et al., 2013; Harrison and Elbrønd, 2018). Trotting may be a particular useful component in warm-up. When trotting, dogs convert potential energy from the ground reaction force into elastic energy stored in tendons and muscles to be released in the stance phase of the trot. This mechanism, also called the 'spring-mass' model (Gregersen et al., 1998), makes the trot a very energy-efficient gait for dogs (Harrison and Elbrønd, 2018). The increase in E-scores, that was found in the TB of the dogs after warm-up, may be a preliminary indication of an improved utilisation of absorbed potential energy as per the 'spring mass' model. For the specific segment, exercises were chosen with the aim of preparing the dog for the rapid weight shifting, coordination and flexibility required in agility. One may argue that jumps should have been included, as short bouts of high intensity exercises have been shown to enhance subsequent performance in human athletes (McGowan et al., 2015). This phenomenon is known as post-activation potentiation (PAP). The potential benefits of PAP exercises in warm-up must be carefully balanced with the risk of fatigue, and factors like training experience may influence the tolerated level of pre-performance loading (McGowan et al., 2015). Higher 
intensity warm-up exercises, such as jumps, may be worth considering for competing canine athletes, but for this trial, with dogs of different training levels and experience, it was not considered appropriate.

It was not possible to document an effect of the duration of warm-up. There was a gradual increase in the E-score with length of warm-up but the differences were not statistically significant. Mean S-score increased from 5 to $10 \mathrm{~min}$ warm-up but not from 10 to $15 \mathrm{~min}$. Longer warm-up times did not seem to cause fatigue, as fatigue would have been visualised as a decrease in the scores (Harrison, 2017). For details of the changes in the E-, Sand $\mathrm{T}$-parameters with fatigue the reader is referred to Harrison (2017). In brief, a non-fatigued muscle exhibits both a stable amplitude (S-score) and frequency (T-score) during a period of sustained physical activity. When fatigue sets in, an irregular signal in terms of amplitude and/or frequency becomes apparent, and with continued physical effort, there is a decrease in the signal amplitude. In a study comparing AMG with surface electromyography, Barry et al. (1985) demonstrated how AMG is superior to surface electromyography for monitoring fatigue. Simultaneous recordings were made from the biceps brachii of healthy adults during isometric contraction. With AMG, which is a measure of muscle fibre contractions, fatigue became readily apparent as an amplitude decay, while the surface electromyography signal, which reflects muscle fibre action potentials, remained unaltered as fatigue set in. In this trial mean amplitude was the same after 10 and 15 min of warmup. However, as it can be seen from Tables 3-5, in some of the dog groups, $\mathrm{S}$-scores were actually slightly lower after $15 \mathrm{~min}$ warm-up compared to after $10 \mathrm{~min}$. These insignificant differences were probably due to random variation, but it cannot be ruled out that individual dogs obtained lower $\mathrm{S}$-scores after $15 \mathrm{~min}$ due to fatigue.

The muscle function parameters were tested for potential differences that could be related to various dog characteristics, and significant results were found in relation to training level, experience and dog height. In the interpretation of the results one must bear in mind that the analyses have low power, as they are based on small and often uneven group sizes and variance was high. Therefore, random fluctuations must be expected. However, consistent tendencies deserve consideration. Dogs that trained for less than $1 \mathrm{~h}$ a week, had lower S-scores for TB than those who trained more. This was seen before warm-up $(2.4 \pm 1.9$ vs $4.7 \pm 1.6, P=0.06)$ and after warm-up, though the difference was only significant after $10 \mathrm{~min}$ and 15 min warm-up (Table 3). For level of experience, an influence on muscle parameters was evident in GS. Dogs with less than two years of experience had consistently lower T-scores (higher firing frequency) than the more experienced dogs, and this difference was significant before warm-up and after 10 min of warm-up. As can be seen from Table 5, the same tendency, although on a smaller scale, was seen for the E- and S-scores. The results support the general concept that experience and frequent training have a positive effect on muscle performance. Other canine agility studies have documented beneficial effects of training at least once a week, as Zachary et al. (2014) found a decreased risk of injury for dogs that trained more than $2 \mathrm{~h}$ a week, and Cullen et al. (2013) found a tendency that dogs that trained less than once a week were at higher risk of injury than dogs that trained more often. It should be mentioned that among the dogs with experience $\geq 2$ years, the mean T-score of GS with any length of warm-up was lower than baseline. This should not be interpreted as a negative effect of warm-up in this group. The explanation probably lies in a very high baseline T-score, which made little room for an increase, and random variation or increasing $\mathrm{S}$-scores could be the reasons for decreasing $\mathrm{T}$-scores.

The height of the dog seemed to have an impact on muscle fibre recruitment of the TB. Here the S-score was consistently higher in shorter dogs compared to taller dogs. But the variance was also high, particularly in the taller group, and the difference was only statistically significant after $15 \mathrm{~min}$ of warm-up. The reason for this difference in amplitude in relation to height is not clear. A similar trend was not seen for the E- or T-scores. It cannot be ruled out that, due to the size of the sensors relative to the muscle width, the signals could be less muscle specific in smaller dogs. It could also be argued that thicker muscles in taller dogs may give rise to a greater signal amplitude. However, this is considered unlikely as there was no tendency of an effect on the muscle parameters based on weight or sex, and as demonstrated by Harrison (2017), AMG measurements of a human biceps brachii muscle showed identical signal amplitude with the sensors placed in various positions overlying more or less muscle bulk. Further studies are required to determine if muscle fibre recruitment in a trotting dog is influenced by the height of the dog.

The dogs had walked a distance of approximately $160 \mathrm{~m}$ from the car to the training area and moved a bit around before the equipment was put on. This level of activity was intentional as it is comparable with real-life training or competition situations, and some owners may regard the walk from the car to the training area as an appropriate warm-up routine. A limitation to this study is that there was no control group with dogs that had been on a leash next to the owner for $15 \mathrm{~min}$ without any specific activities. Another limitation concerns the risk of variations in movement, when the dogs trotted next to the owner during the 4 recordings. Ideally, measures should have been taken to ensure stable speed and posture. However, it is considered unlikely that this lack of standardisation has influenced the results, as the dogs were found to trot at a natural pace and care was taken to perform this activity as uniformly as possible. 
The implementation of well-designed regular warm-up protocols are believed to reduce risk of injury among human athletes (Racinais et al., 2017; Woods et al., 2007). Two retrospective studies have not found a link between warmup and risk of injury in dogs (Cullen et al., 2013; Zachary et al., 2014). However, the results could be biased, as these studies were based on owner reports, and 'warm-up' was not specified in respect to duration or type of exercise. The present study serves as a preliminary indication that warm-up improves muscle function, and therefore one might speculate that warm-up may enhance performance and reduce the risk of injury in agility dogs and probably other short-duration high-intensity canine sports.

\section{Conclusions}

Acoustic myography can be used to gain information of muscle activation in dogs under natural conditions. A warm-up program increased TB efficiency, and thus, the results support the recommendation of the inclusion of a warm-up routine in canine sports. Further studies are required to determine if a 10 or $15 \mathrm{~min}$ program may be more effective than a $5 \mathrm{~min}$ program. Among the dogs included in this program, muscle fibre recruitment and firing frequency were influenced by training level and experience.

\section{Acknowledgements}

The authors would like to thank the dog owners for giving of their time and participating in this study. A special thanks is also due to Kiara K. Salomons Heide for assisting mounting the AMG equipment and aid during the subsequent recordings. Finally, the authors also wish to thank Dr Vibeke Sødring Elbrønd for assisting with her expert knowledge of the anatomy and physiology of the canine muscles and the positioning of the AMG sensors.

\section{Conflict of interest}

$\mathrm{AH}$ is in the process of forming a company with the aim of commercialising the Acoustic Myography equipment. These results have been published as a 250 -word abstract at the ECVSMR/VEPRA Conference, September 2019, Ghent, Belgium

\section{Supplementary material}

Supplementary material can be found online at https://doi. org/10.3920/CEP190076.

Table S1. Raw data.

Table S2. Gender difference.
Table S4. Weight.

Table S5. Age.

Table S6. Experience.

Table S7. Training level.

\section{References}

Alves, N. and Chau, T., 2011. Mechanomyography as an access pathway: corporeal contraindications. Disability and Rehabilitation Assistive Technology 6: 552-563. https://doi.org/10.3109/174831 07.2010 .541323

Barry, D.T., Geiringer, S.R. and Ball, R.D., 1985. Acoustic myography: a noninvasive monitor of motor unit fatigue. Muscle and Nerve 8: 189-194.

Barry, D.T., Gordon, K.E. and Hinton, G.G., 1990. Acoustic and surface EMG diagnosis of pediatric muscle disease. Muscle and Nerve 13: 286-290.

Bartels, E.M., Harder, A., Heide, K.S., Pingel, J., Andersen, I.T. and Harrison, A.P., 2017. The use of acoustic myography as a measure of training effects in athletes - a 10 month case study of a BMX rider. Annals of Sport Medicine and Research 4: 1101.

Beck, T.W., Defreitas, J.M., Stock, M.S. and Dillon, M.A., 2010. An explanation of mechanomyographic signal stationarity during concentric isokinetic, eccentric isokinetic and isometric muscle actions. Physiological Measurement 31: 339-361. https://doi. org/10.1088/0967-3334/31/3/005

Bishop, D., 2003. Warm up I: potential mechanisms and the effects of passive warm up on exercise performance. Sports Medicine 33: 439-454. https://doi.org/10.2165/00007256-200333060-00005

Carvalhais, V.O., Oscarino, J., Araújo, V.L., Souza, T.R., Silva, P.L. and Fonseca, S.T., 2013. Myofascial force transmission between the latissimus dorsi and gluteus maximus muscles: an in vivo experiment. Journal of Biomechanics 46: 1003-1007. https://doi. org/10.1016/j.jbiomech.2012.11.044

Cullen, K.L., Dickey, J.P., Bent, L.R., Thomason, J.J. and Moëns, N.M.M., 2013. Survey-based analysis of risk factors for injury among dogs participating in agility training and competition events. Journal of the American Veterinary Medical Association 243: 1019-1024. https://doi.org/10.2460/javma.243.7.1019

Deban, S.M., Schilling, N. and Carrier, D.R., 2012. Activity of extrinsic limb muscles in dogs at walk, trot and gallop. Journal of Experimental Biology 215: 287-300. https://doi.org/10.1242/jeb.063230

Evans, H.E. and De Lahunta, A., 2000. Guide to the dissection of the dog, $5^{\text {th }}$ edition. Saunders, London, UK, pp. 78.Faigenbaum, A.D, Bellucci, M., Bernieri, A., Bakker, B. and Hoorens, K., 2005. Acute effects of different warm-up protocols on fitness performance in children. Journal of Strength and Conditioning Research 19: 376-381.

Federation Cynologique Internationale, 2018. Federation Cynologique Internationale agility regulations. Federation Cynologique Internationale, Thuin, Belgium. Available at: http://fci.be/en/ Agility-45.html

Table S3. Height. 
Fenger, C. and Harrison, A.P., 2017. The application of acoustic myography in canine muscle function and performance testing. Symbiosis Journal of Veterinary Science 3: 1-6. https://doi. org/10.15226/2381-2907/3/2/00128

Fradkin, A.J., Zazryn, T.S. and Smoliga, J.M., 2010. Effects of warmingup on physical performance: a systematic review with meta-analysis. Journal of Strength and Conditioning Research 24: 140-148. https:// doi.org/10.1519/JSC.0b013e3181c643a0

Gregersen, C.S., Silverton, N.A. and Carrier, D.R., 1998. External work and potential for elastic storage at the limb joints of running dogs. Journal of Experimental Biology 201: 3197-3210.

Harrison, A.P. and Elbrønd, V.S., 2018. Applied myo-fascial advances in veterinary medicine and practice. Science Publishing Group BioMed 2018: 24. https://doi.org/10.32392/biomed.24.1

Harrison, A.P., 2017. A more precise, repeatable and diagnostic alternative to surface electromyography - an appraisal of the clinical utility of acoustic myography. Clinical Physiology and Functional Imaging 38: 312-325. https://doi.org/10.1111/cpf.12417

Harrison, A.P., Danneskiold-Samsøe, B. and Bartels, E.M., 2013. Portable acoustic myography - a realistic noninvasive method for assessment of muscle activity and coordination in human subjects in most home and sports settings. Physiological Reports 1: 1-9. https://doi.org/10.1002/phy2.29

Harrison, A.P., Jensen, A., Riis, K.A. and Riis-Olsen, K., 2018. Noninvasive assessment of lameness in horses with dorsal spinous process impingement 'Kissing spine': a case study. Multidisciplinary Advances in Veterinary Science 1: 257-265.

Hemmerling, T.M., Michaud, G., Trager, G., Deschamps, S., Babin, D. and Donati, F., 2004. Phonomyography and mechanomyography can be used interchangeably to measure neuromuscular block at the adductor pollicis muscle. Anesthesia and Analgesia 98: 377-381. https://doi.org/10.1213/01.ane.0000096003.64059.97

Herda, T.J., Housh, T.J., Fry, A.C., Weir, J.P., Schilling, B.K., Ryan, E.D. and Cramer, J.T., 2010. A noninvasive, log-transform method for fiber type discrimination using mechanomyography. Journal of Electromyography and Kinesiology 20: 787-794. https://doi. org/10.1016/j.jelekin.2010.01.004

Kilduff, L.P., Finn, C.V., Baker, J.S., Cook, C.J. and West, D.J., 2013. Preconditioning strategies to enhance physical performance on the day of competition. International Journal of Sports Physiology and Performance 8: 677-681. https://doi.org/10.1123/ijspp.8.6.677

Laflamme, D., 1997. Development and validation of a body condition score system for dogs. Canine Practice 22: 10-15.

Lafuente, P. and Whyle, C., 2018. A retrospective survey of injuries occurring in dogs and handlers participating in canicross. Veterinary and Comparative Orthopaedics and Traumatology 31: 332-338. https://doi.org/10.1055/s-0038-1661390

Levy, I., Hall, C. and Percival, M., 2009. A preliminary retrospective survey of injuries occurring in dogs participating in canine agility. Veterinary and Comparative Orthopaedics and Traumatology 22: 321-324. https://doi.org/10.3415/VCOT-08-09-0089

Madeleine, P. and Arendt-Nielsen, L., 2005. Experimental muscle pain increases mechanomyographic signal activity during submaximal isometric contractions. Journal of Electromyography and Kinesiology 15: 27-36. https://doi.org/10.1016/j.jelekin.2004.06.006
McGowan, C.J., Pyne, D.B., Thompson, K.G. and Rattray, B., 2015. Warm-up strategies for sport and exercise: mechanisms and applications. Sports Medicine 45: 1523-1546. https://doi. org/10.1007/s40279-015-0376-x

Perry, S.R., Housh, T.J., Weir, J.P., Johnson, G.O., Bull, A.J. and Ebersole, K.T., 2001. Mean power frequency and amplitude of the mechanomyographic and electromyographic signals during incremental cycle ergometry. Journal of Electromyography and Kinesiology 11: 299-305. https://doi.org/10.1016/s10506411(00)00057-2

Petrofsky, J.S., Laymon, M. and Haneul, L., 2013. Effect of heat and cold on tendon flexibility and force to flex the human knee. Medical Science Monitor 19: 661-667. https://doi.org/10.12659/MSM.889145

Pingel, J., Andersen, I.T., Broholm, R., Harder, A., Bartels, E.M., Bülow, J. and Harrison, A., 2019. An acoustic myography functional assessment of cerebral palsy subjects compared to healthy controls during physical exercise. Journal of Muscle Research and Cell Motility 40: 53-58. https://doi.org/10.1007/s10974-019-09516-y

Qi, L., Wakeling, J.M., Green, A., Lambrecht, K. and Ferguson-Pell, M., 2011. Spectral properties of electromyographic and mechanographic signals during isometric ramp and step contractions in biceps brachii. Journal of Electromyography and Kinesiology 22: 128-135. https://doi.org/10.1016/j.jelekin.2010.09.006

Racinais, S., Cocking, S. and Périard, J.D., 2017. Sports and environmental temperature: from warming-up to heating-up. Temperature 4: 227-257. https://doi.org/10.1080/23328940.201 7.1356427

Riis, K.H., Harrison, A.P. and Riis-Olesen, K., 2013. Non-invasive assessment of equine muscular function: a case study. Open Veterinary Journal 3: 80-84.

Schilling, N., Fischbein, T., Yand, E.P. and Carrier, D.R., 2009. Function of the extrinsic hindlimb muscles in trotting dogs. Journal of Experimental Biology 212: 1036-1052.

Shinohara, M. and Søgaard, K., 2006. Mechanomyography for studying force fluctuations and muscle fatigue. Exercise and Sport Science Reviews 34: 59-64. https://doi.org/10.1249/00003677-20060400000004

Stokes, M. and Blythe, M., 2001. Muscle sounds in physiology, sports science and clinical investigation. Medintel - Medical Intelligence Ltd., Oxford, UK.

Strickler, T., Malone, T. and Garrett, W.E., 1990. The effects of passive warming on muscle injury. American Journal of Sports Medicine 18: 141-145. https://doi.org/10.1177/036354659001800206

Tian, S.L., Liu, Y., Li, L., Fu, W.J. and Peng, C.H., 2010. Mechanomyography is more sensitive than EMG in detecting age-related sarcopenia. Journal of Biomechanics 43: 551-556. https://doi. org/10.1016/j.jbiomech.2009.09.034

Tsolakis, C. and Bogdanis, G.C., 2012. Acute effects of two different warm-up protocols on flexibility and lower limb explosive performance in male and female high level athletes. Journal of Sports Science and Medicine 11: 669-675

Williams, S.B., Wilson, A.M., Rhodes, L., Andrews, J. and Payne, R.C., 2008. Functional anatomy and muscle moment arms of the thoracic limb of an elite sprinting athlete: the racing greyhound (Canis familiaris). Journal of Anatomy 213: 372-382. https://doi. org/10.1111/j.1469-7580.2008.00962.x 
Woods, K., Bishop, P. and Jones, E., 2007. Warm-up and stretching in the prevention of muscular injury. Sports Medicine 37: 1089-1099. https://doi.org/10.2165/00007256-200737120-00006

Youn, W. and Kim, J., 2010. Estimation of elbow flexion force during isometric muscle contraction from mechanomyography and electromyography. Medical and Biological Engineering and Computing 48: 1149-1157. https://doi.org/10.1007/s11517-0100641-y
Zachary, Y.K., Fields, S. and Comstock, R.D., 2014. Epidemiology of injury among handlers and dogs competing in the sport of agility. Journal of Physical Activity and Health 11: 1032-1040. https://doi. org/10.1123/jpah.2012-0236 